\title{
Measure of non-compactness of operators interpolated by the real method
}

\author{
by \\ RadosŁaW Szwedek (Poznań)
}

\begin{abstract}
We study the measure of non-compactness of operators between abstract real interpolation spaces. We prove an estimate of this measure, depending on the fundamental function of the space. An application to the spectral theory of linear operators is presented.
\end{abstract}

1. Introduction. The behavior of compact linear operators under interpolation has been studied since the 1960s. First results were established by Krasnosel'skiı [11], who proved that under the hypothesis of the RieszThorin interpolation theorem, that is, $T: L_{p_{i}} \rightarrow L_{q_{i}}$ is bounded for $i=0,1$ where $1 \leq p_{i}, q_{i} \leq \infty$, and the additional assumption that $T: L_{p_{0}} \rightarrow L_{q_{0}}$ is compact, $q_{0}<\infty$, it follows that $T: L_{p} \rightarrow L_{q}$ is also compact, where $1 / p=(1-\theta) / p_{0}+\theta / p_{1}, 1 / q=(1-\theta) / q_{0}+\theta / q_{1}$ and $0<\theta<1$.

His results lead to the question whether similar results hold in the abstract interpolation case, when $\left(L_{p_{0}}, L_{p_{1}}\right)$ and $\left(L_{q_{0}}, L_{q_{1}}\right)$ are replaced by Banach pairs $\left(A_{0}, A_{1}\right)$ and $\left(B_{0}, B_{1}\right)$, respectively. The complete answer is still unknown.

The first results for the real interpolation method were obtained in 1964 by Lions-Peetre [12] for the case when $A_{0}=A_{1}$ or $B_{0}=B_{1}$ and by Persson [14] for the general case $A_{0} \neq A_{1}$ and $B_{0} \neq B_{1}$ with an approximation condition on the couple $\left(B_{0}, B_{1}\right)$. In these cases, they showed that the operator $T: \bar{A}_{\theta, q} \rightarrow \bar{B}_{\theta, q}$ is compact for $0<\theta<1,1 \leq q \leq \infty$. We refer to the book [2] for a more detailed history of research.

In 1969 Hayakawa [10] gave the result for the real interpolation method without the approximation hypothesis but with the assumption that $T$ : $\left(A_{0}, A_{1}\right) \rightarrow\left(B_{0}, B_{1}\right)$ and the restrictions $T: A_{0} \rightarrow B_{0}, T: A_{1} \rightarrow B_{1}$ are both compact operators and $1 \leq q<\infty$. New approaches to Hayakawa's result can be found in the paper by Cobos and Peetre [7] and the references given

2000 Mathematics Subject Classification: 46B70, 46M35.

Key words and phrases: linear operators, measure of non-compactness, interpolation. 
there. Finally, in 1992 Cwikel [9] showed that the theorem holds whenever $T: A_{0} \rightarrow B_{0}$ is compact and $T: A_{1} \rightarrow B_{1}$ is bounded.

Nowadays we search for quantitative versions of interpolating compactness results. In 1999, Cobos, Fernández-Martínez and Martínez in their remarkable paper [6] obtained a logarithmic type inequality for the measure of non-compactness $\beta(\cdot)$, namely

$$
\beta\left(T: \bar{A}_{\theta, q} \rightarrow \bar{B}_{\theta, q}\right) \leq C \beta\left(T: A_{0} \rightarrow B_{0}\right)^{1-\theta} \beta\left(T: A_{1} \rightarrow B_{1}\right)^{\theta} .
$$

Based on some ideas from [6], we present a generalization of those results to the abstract real method of interpolation.

2. Preliminaries and notation. We use the standard notation from interpolation theory. Mostly, we follow [2] and [3], where more details are given. Let $\bar{A}=\left(A_{0}, A_{1}\right)$ be a Banach couple. As usual we let $\Delta(\bar{A}):=A_{0} \cap A_{1}$ and $\Sigma(\bar{A}):=A_{0}+A_{1}$. For any $t>0$ the $K$-functional is defined by

$$
K(t, a ; \bar{A})=\inf _{a=a_{0}+a_{1}}\left\{\left\|a_{0}\right\|_{A_{0}}+t\left\|a_{1}\right\|_{A_{1}}\right\} \quad \text { for } a \in \Sigma(\bar{A}),
$$

and the $J$-functional by

$$
J(t, a ; \bar{A})=\max \left\{\|a\|_{A_{0}}, t\|a\|_{A_{1}}\right\} \quad \text { for } a \in \Delta(\bar{A}) .
$$

A real sequence $w=\left\{w_{n}\right\}_{n \in \mathbb{Z}}$ is called a weight sequence if each $w_{n}$ is positive. If $E$ is a Banach sequence lattice modelled on $\mathbb{Z}$ and $w=\left\{w_{n}\right\}$ is a weight sequence, we define the weighted Banach sequence lattice $E(w)$ by setting $\|x\|_{E(w)}:=\left\|\left\{x_{n} w_{n}\right\}\right\|_{E}$.

Recall that if a Banach sequence lattice $E$ on $\mathbb{Z}$ is intermediate with respect to $\left(\ell_{\infty}, \ell_{\infty}\left(2^{-n}\right)\right)$ (resp., $\left.\left(\ell_{1}, \ell_{1}\left(2^{-n}\right)\right)\right)$ then the $K$-method space $\bar{A}_{E ; K}:=\left(A_{0}, A_{1}\right)_{E ; K}$ consists of all $a \in A_{0}+A_{1}$ such that $\left\{K\left(2^{n}, a ; \bar{A}\right)\right\} \in E$ with the norm

$$
\|a\|=\left\|\left\{K\left(2^{n}, a ; \bar{A}\right)\right\}\right\|_{E}
$$

while the $J$-method space $\bar{A}_{E ; J}$ consists of all $a \in A_{0}+A_{1}$ which can be represented in the form

$$
\left.a=\sum_{n=-\infty}^{\infty} a_{n} \quad \text { (convergence in } A_{0}+A_{1}\right)
$$

such that $\left\{J\left(2^{n}, a_{n} ; \bar{A}\right)\right\}_{n \in \mathbb{Z}} \in E$ with the associated norm

$$
\|a\|=\inf \left\{\left\|\left\{J\left(2^{n}, a_{n} ; \bar{A}\right)\right\}\right\|_{E}: a=\sum_{n=-\infty}^{\infty} a_{n}\right\} .
$$

Following the terminology of [13], the space $E$ is said to be $K$-non-trivial (resp., J-non-trivial) when $\ell_{\infty} \cap \ell_{\infty}\left(2^{-n}\right) \subset E$ (resp., $\left.E \subset \ell_{1}+\ell_{1}\left(2^{-n}\right)\right)$. 
It is well known from [13] that if $E$ is a parameter of the real method (i.e., $\left.\ell_{\infty} \cap \ell_{\infty}\left(2^{-n}\right) \subset E \subset \ell_{1}+\ell_{1}\left(2^{-n}\right)\right)$ then for any Banach couple $\bar{A}$,

$$
\bar{A}_{E ; K} \hookrightarrow \bar{A}_{E ; J}
$$

and the norm of the inclusion map is less than 4 . If additionally the Calderón operator $\Omega$ defined on $\ell_{1}+\ell_{1}\left(2^{-n}\right)$ by

$$
\Omega\left\{\xi_{n}\right\}:=\left\{\sum_{k=-\infty}^{\infty} \min \left\{1,2^{n-k}\right\}\left|\xi_{k}\right|\right\} \quad \text { for }\left\{\xi_{n}\right\} \in \ell_{1}+\ell_{1}\left(2^{-n}\right)
$$

is bounded on $E$, then $\bar{A}_{E ; J}=\bar{A}_{E ; K}$. In this case we write $\bar{A}_{E}$ for $\bar{A}_{E ; J}$ or $\bar{A}_{E ; K}$.

The classical interpolation spaces play an important role from the point of view of applications. Let $\varrho$ be a function parameter, i.e., $\varrho:(0, \infty) \rightarrow(0, \infty)$ is a quasi-concave function $(t \mapsto \varrho(t)$ increases and $t \mapsto \varrho(t) / t$ decreases $)$ and

$$
s_{\varrho}(t)=o(1) \text { as } t \rightarrow 0 \text { and } s_{\varrho}(t)=o(t) \text { as } t \rightarrow \infty,
$$

where $s_{\varrho}(t)=\sup \{\varrho(t u) / \varrho(u): u>0\}$ for every $t>0$. Now, if we take $E=\ell_{q}\left(1 / \varrho\left(2^{m}\right)\right)$ with $1 \leq q \leq \infty$, then

$$
\left(A_{0}, A_{1}\right)_{\ell_{q}\left(1 / \varrho\left(2^{m}\right)\right) ; K}=\left(A_{0}, A_{1}\right)_{\ell_{q}\left(1 / \varrho\left(2^{m}\right)\right) ; J}=\left(A_{0}, A_{1}\right)_{\varrho, q} .
$$

If $\varrho(t)=t^{\theta}, \theta \in(0,1)$, we get the classical real interpolation spaces $\left(A_{0}, A_{1}\right)_{\theta, q}$ (see, e.g., [2], [3]).

Given any sequence $\left\{X_{m}\right\}_{m \in \mathbb{Z}}$ of Banach spaces we denote by $\left(\bigoplus X_{m}\right)_{E}$ the space of $\left\{x_{m}\right\}_{m \in \mathbb{Z}} \in \prod_{m=-\infty}^{\infty} X_{m}$ such that $\left\{\left\|x_{m}\right\|_{X_{m}}\right\}_{m \in \mathbb{Z}} \in E$. It is a Banach space equipped with the norm

$$
\left\|\left\{x_{m}\right\}\right\|=\left\|\left\{\left\|x_{m}\right\|_{X_{m}}\right\}\right\|_{E}
$$

We will need the following useful vector-valued continuous inclusions (with norms less than or equal to 1) proved in [5]:

Proposition 2.1. Let $\left\{X_{m}\right\}_{m \in \mathbb{Z}}$ be a sequence of Banach spaces.

(i) If $E$ is $K$-non-trivial then

$$
\left(\left(\bigoplus X_{m}\right)_{\ell_{\infty}},\left(\bigoplus X_{m}\right)_{\ell_{\infty}\left(2^{-m}\right)}\right)_{E ; K} \hookrightarrow\left(\bigoplus X_{m}\right)_{E} .
$$

(ii) If $E$ is J-non-trivial then

$$
\left(\bigoplus X_{m}\right)_{E} \hookrightarrow\left(\left(\bigoplus X_{m}\right)_{\ell_{1}},\left(\bigoplus X_{m}\right)_{\ell_{1}\left(2^{-m}\right)}\right)_{E ; J}
$$

An immediate consequence is

Corollary 2.2. Let $\left\{X_{m}\right\}_{m \in \mathbb{Z}}$ be a sequence of Banach spaces. If the $J$-method and the $K$-method generated by a parameter $E$ of the real method are equivalent then

$$
\left(\left(\bigoplus X_{m}\right)_{\ell_{q_{0}}},\left(\bigoplus X_{m}\right)_{\ell_{q_{1}}\left(2^{-m}\right)}\right)_{E}=\left(\bigoplus X_{m}\right)_{E}
$$

for any $1 \leq q_{j} \leq \infty(j=0,1)$. 
For a given Banach lattice $E$ on $\mathbb{Z}$ and a subset $A \subset \mathbb{Z}$, the subspace of all sequences supported on $A$ is denoted by $\left.E\right|_{A}$. In particular, we may take $A$ to be $[p, q]:=\{n \in \mathbb{Z}: p \leq n \leq q\}$ with $p, q \in \mathbb{Z}, p<q$.

Let $\omega(\mathbb{Z})$ be the space of all real sequences modelled on $\mathbb{Z}$. For any $\nu \in \mathbb{Z}$, the shift operator $\tau_{\nu}: \omega(\mathbb{Z}) \rightarrow \omega(\mathbb{Z})$ is defined by $\tau_{\nu}\left\{\xi_{m}\right\}=\left\{\xi_{m+\nu}\right\}$.

Throughout the rest of the paper we consider Banach lattices $E$ on $\mathbb{Z}$ such that the shift operator $\tau_{\nu}$ is bounded in $E$ for all $\nu \in \mathbb{Z}$. For such $E$ we define a function $\varphi_{E}:(0, \infty) \times(0, \infty) \rightarrow(0, \infty)$ by $\varphi_{E}\left(2^{m}, 2^{n}\right)=2^{m}\left\|\tau_{n-m}\right\|_{E \rightarrow E}$ for $m, n \in \mathbb{Z}$ and $\varphi_{E}(s, t)=\varphi_{E}\left(2^{\left[\log _{2} s\right]}, 2^{\left[\log _{2} t\right]}\right)$ for any $s, t>0$, where $[\cdot]$ denotes the greatest integer function.

We will also need the extension $\psi_{E}:[0, \infty) \times[0, \infty) \rightarrow[0, \infty]$ of $\varphi_{E}$, defined by $\psi_{E}(0,0)=0, \psi_{E}(s, 0)=\liminf _{v \rightarrow 0+} \varphi_{E}(s, v)$ for $s>0$ and $\psi_{E}(0, t)=\liminf _{u \rightarrow 0+} \varphi_{E}(u, t)$ for $t>0$.

We have the following technical lemma:

LEMMA 2.3. Let $E$ be a Banach sequence lattice on $\mathbb{Z}$ such that the shift operator $\tau_{n}$ is bounded in $E$ for any $n \in \mathbb{N}$. Then the function $\psi_{E}$ has the following properties:

(a) $\psi_{E}\left(2^{m} s, 2^{n} t\right) \leq \psi_{E}\left(2^{m}, 2^{n}\right) \psi_{E}(s, t)$ for all $m, n \in \mathbb{Z}$ and $s, t \geq 0$.

(b) There exists a constant $C_{1}=C_{1}(E) \geq 1$ such that

$$
\psi_{E}(s u, t v) \leq C_{1} \psi_{E}(s, t) \psi_{E}(u, v) \quad \text { for all } s, t, u, v \geq 0 .
$$

(c) If $\sup _{s, t \in(0,1]} \psi_{E}(s, t)<\infty$, then there exists a constant $C_{2}=C_{2}(E)$ $\geq 1$ such that

$$
\psi_{E}(s, t) \leq C_{2} \psi_{E}(u, v) \quad \text { for all } 0 \leq s \leq u, 0 \leq t \leq v .
$$

Proof. Since $\psi_{E}\left(2^{m+k}, 2^{n+l}\right) \leq \psi_{E}\left(2^{m}, 2^{n}\right) \psi_{E}\left(2^{k}, 2^{l}\right)$ and

$$
\begin{aligned}
{\left[\log _{2} 2^{k} s\right] } & =k+\left[\log _{2} s\right], \\
{\left[\log _{2} s\right]+\left[\log _{2} t\right] } & \leq\left[\log _{2} s t\right] \leq\left[\log _{2} s\right]+\left[\log _{2} t\right]+1,
\end{aligned}
$$

for any $m, k, n, l \in \mathbb{Z}$ and $s, t>0$, we get (a) and (b) with a constant $C_{1}=\max _{i, j=0,1} \psi_{E}\left(2^{i}, 2^{j}\right)$.

For $(\mathrm{c})$, since $\sup _{s, t \in(0,1]} \psi_{E}(s, t)<\infty$, using the previously proved inequalities, we obtain

$$
\psi_{E}(s, t)=\psi_{E}\left(\frac{s}{u} u, \frac{t}{v} v\right) \leq C_{2} \psi_{E}(u, v)
$$

for all $0<s \leq u, 0<t \leq v$ where $C_{2}=C_{1} \sup _{s, t \in(0,1]} \psi_{E}(s, t)<\infty$. Recalling that $\psi_{E}(0, t)=\liminf _{s \rightarrow 0+} \psi_{E}(s, t)$ and $\psi_{E}(s, 0)=\liminf _{t \rightarrow 0+} \psi_{E}(s, t)$ completes the proof.

LEMma 2.4. Let $\bar{A}=\left(A_{0}, A_{1}\right)$ and $\bar{B}=\left(B_{0}, B_{1}\right)$ be Banach couples and let $T: \bar{A} \rightarrow \bar{B}$ be a bounded operator. 
(i) If $E$ is K-non-trivial, then

$$
\|T\|_{\bar{A}_{E ; K} \rightarrow \bar{B}_{E ; K}} \leq 2 \psi_{E}\left(\|T\|_{A_{0} \rightarrow B_{0}},\|T\|_{A_{1} \rightarrow B_{1}}\right) .
$$

(ii) If $E$ is J-non-trivial, then

$$
\|T\|_{\bar{A}_{E ; J} \rightarrow \bar{B}_{E ; J}} \leq 2 \psi_{E}\left(\|T\|_{A_{0} \rightarrow B_{0}},\|T\|_{A_{1} \rightarrow B_{1}}\right) .
$$

Proof. (i) Since the case $\|T\|_{A_{0} \rightarrow B_{0}}=\|T\|_{A_{1} \rightarrow B_{1}}=0$ is obvious, we may assume that $\|T\|_{A_{i} \rightarrow B_{i}}>0$ for $i=0$ or $i=1$. Fix $\varepsilon, \delta \geq 0$ with $\varepsilon=0$ if and only if $\|T\|_{A_{0} \rightarrow B_{0}}>0$, and $\delta=0$ if and only if $\|T\|_{A_{1} \rightarrow B_{1}}>0$. Now take $k_{0}, k_{1} \in \mathbb{Z}$ such that $2^{k_{0}-1} \leq\|T\|_{A_{0} \rightarrow B_{0}}+\varepsilon<2^{k_{0}}$ and $2^{k_{1}-1} \leq$ $\|T\|_{A_{1} \rightarrow B_{1}}+\delta<2^{k_{1}}$. Let $\nu=k_{1}-k_{0}$. Then for any $a \in \bar{A}_{E ; K}$ we have

$$
\begin{aligned}
K\left(2^{m}, T a ; \bar{B}\right) & \leq \inf _{a=a_{0}+a_{1}}\left\{\left\|T a_{0}\right\|_{B_{0}}+2^{m}\left\|T a_{1}\right\|_{B_{1}}: a_{0} \in A_{0}, a_{1} \in A_{1}\right\} \\
& \leq \inf _{a=a_{0}+a_{1}}\left\{2^{k_{0}}\left\|a_{0}\right\|_{A_{0}}+2^{m+k_{1}}\left\|a_{1}\right\|_{A_{1}}: a_{0} \in A_{0}, a_{1} \in A_{1}\right\} \\
& =2^{k_{0}} K\left(2^{m+\nu}, a ; \bar{A}\right),
\end{aligned}
$$

and hence

$$
\begin{aligned}
\|T a\|_{\bar{B}_{E ; K}} & =\left\|\left\{K\left(2^{m}, T a ; \bar{B}\right)\right\}\right\|_{E} \leq 2^{k_{0}}\left\|\left\{K\left(2^{m+\nu}, a ; \bar{A}\right)\right\}\right\|_{E} \\
& =2^{k_{0}}\left\|\tau_{\nu}\left\{K\left(2^{m}, a ; \bar{A}\right)\right\}\right\|_{E} \leq 2^{k_{0}}\left\|\tau_{\nu}\right\|_{E \rightarrow E}\left\|\left\{K\left(2^{m}, a ; \bar{A}\right)\right\}\right\|_{E} \\
& =2^{1+k_{0}-1}\left\|\tau_{k_{1}-1-\left(k_{0}-1\right)}\right\|\left\|_{E \rightarrow E}\right\| a\left\|_{\bar{A}_{E ; K}}=2 \varphi_{E}\left(2^{k_{0}-1}, 2^{k_{1}-1}\right)\right\| a \|_{\bar{A}_{E ; K}} \\
& =2 \varphi_{E}\left(\|T\|_{A_{0} \rightarrow B_{0}}+\varepsilon,\|T\|_{A_{1} \rightarrow B_{1}}+\delta\right)\|a\|_{\bar{A}_{E ; K}} .
\end{aligned}
$$

If $\varepsilon, \delta=0$ then the above estimate gives the required inequality. If either $\varepsilon>0$ or $\delta>0$ then the inequality follows by taking the relevant limits. This completes the proof of (i).

(ii) Let $a \in \bar{A}_{E ; J}$ and take any series $\sum_{m=-\infty}^{\infty} u_{m}$ convergent in $A_{0}+A_{1}$ to $a$, where $\left\{u_{m}\right\} \subset A_{0} \cap A_{1}$ is such that $\left\{J\left(2^{m}, u_{m}\right)\right\} \in E$. Since $E$ is $J$-nontrivial, the series $\sum_{m=-\infty}^{\infty} u_{m}$ is absolutely summable in $A_{0}+A_{1}$. Therefore, for any $\nu \in \mathbb{Z}$ we have $a=\sum_{m=-\infty}^{\infty} u_{m+\nu}$ (convergence in $A_{0}+A_{1}$ ) and

$$
\begin{aligned}
J\left(2^{m}, T u_{m+\nu} ; \bar{B}\right) & \leq \max \left\{2^{k_{0}}\left\|u_{m+\nu}\right\|_{A_{0}}, 2^{m+k_{1}}\left\|u_{m+\nu}\right\|_{A_{1}}\right\} \\
& =2^{k_{0}} J\left(2^{m+\nu}, u_{m+\nu} ; \bar{A}\right) .
\end{aligned}
$$

This implies

$$
\begin{aligned}
\|T a\|_{\bar{B}_{E ; J}} & \leq\left\|\left\{J\left(2^{m}, T u_{m+\nu} ; \bar{B}\right)\right\}\right\|_{E} \leq 2^{k_{0}}\left\|\left\{J\left(2^{m+\nu}, u_{m+\nu} ; \bar{A}\right)\right\}\right\|_{E} \\
& =2^{k_{0}}\left\|\tau_{\nu}\left\{J\left(2^{m}, u_{m} ; \bar{A}\right)\right\}\right\|_{E} \leq 2^{k_{0}}\left\|\tau_{\nu}\right\|_{E \rightarrow E}\left\|\left\{J\left(2^{m}, u_{m} ; \bar{A}\right)\right\}\right\|_{E} \\
& =2^{1+k_{0}-1}\left\|\tau_{k_{1}-1-\left(k_{0}-1\right)}\right\|_{E \rightarrow E}\left\|\left\{J\left(2^{m}, u_{m} ; \bar{A}\right)\right\}\right\|_{E} .
\end{aligned}
$$


Taking the infimum over all representations $a=\sum_{m=-\infty}^{\infty} u_{m}$ of $a$, we get

$$
\begin{aligned}
\|T a\|_{\bar{B}_{E ; J}} & \leq 2^{1+k_{0}-1}\left\|\tau_{k_{1}-1-\left(k_{0}-1\right)}\right\|_{E \rightarrow E}\|a\|_{\bar{A}_{E ; J}} \\
& =2 \varphi_{E}\left(\|T\|_{A_{0} \rightarrow B_{0}}+\varepsilon,\|T\|_{A_{1} \rightarrow B_{1}}+\delta\right)\|a\|_{\bar{A}_{E ; J}},
\end{aligned}
$$

which completes the proof as above.

Corollary 2.5. Let $\left\{X_{m}\right\}$ and $\left\{Y_{m}\right\}$ be sequences of Banach spaces, let $E$ be a parameter of the real method, and assume that the Calderón operator $\Omega$ is bounded on $E$. Then there exists a constant $C=C(E)>0$ such that for any bounded operator

$$
T:\left(\left(\bigoplus X_{m}\right)_{\ell_{1}},\left(\bigoplus X_{m}\right)_{\ell_{1}\left(2^{-m}\right)}\right) \rightarrow\left(\left(\bigoplus Y_{m}\right)_{\ell_{\infty}},\left(\bigoplus Y_{m}\right)_{\ell_{\infty}\left(2^{-m}\right)}\right),
$$

we have

$$
\begin{aligned}
\|T\|_{\left(\oplus X_{m}\right)_{E}} \rightarrow\left(\bigoplus Y_{m}\right)_{E} & \\
& \leq C \psi_{E}\left(\|T\|_{\left(\oplus X_{m}\right)_{\ell_{1}} \rightarrow\left(\oplus Y_{m}\right)_{\ell_{\infty}}},\|T\|_{\left.\left(\oplus X_{m}\right)_{\ell_{1}\left(2^{-m}\right)} \rightarrow\left(\oplus Y_{m}\right)_{\ell_{\infty}\left(2^{-m}\right)}\right) .}\right.
\end{aligned}
$$

Proof. Use Proposition 2.1 and Lemma 2.4.

Let $\bar{A}=\left(A_{0}, A_{1}\right)$ be a Banach couple. For $m \in \mathbb{Z}$ define the Banach spaces

$$
\begin{aligned}
\Delta_{m}(\bar{A}) & =\left(A_{0} \cap A_{1}, J\left(2^{m}, \cdot ; \bar{A}\right)\right), \\
\Sigma_{m}(\bar{A}) & =\left(A_{0}+A_{1}, K\left(2^{m}, \cdot ; \bar{A}\right)\right) .
\end{aligned}
$$

We note that for any sequence

$$
\left\{a_{n}\right\} \in\left(\bigoplus \Delta_{m}(\bar{A})\right)_{\ell_{1}}+\left(\bigoplus \Delta_{m}(\bar{A})\right)_{\ell_{1}\left(2^{-m}\right)}=\left(\bigoplus \Delta_{m}(\bar{A})\right)_{\ell_{1}+\ell_{1}\left(2^{-m}\right)}
$$

the series $\sum_{n=-\infty}^{\infty} a_{n}$ is absolutely convergent in $A_{0}+A_{1}$. This allows us to define a bounded operator $\pi:\left(\left(\bigoplus \Delta_{m}(\bar{A})\right)_{\ell_{1}},\left(\bigoplus \Delta_{m}(\bar{A})\right)_{\ell_{1}\left(2^{-m}\right)}\right) \rightarrow\left(A_{0}, A_{1}\right)$ by

$$
\pi\left\{a_{n}\right\}=\sum_{n=-\infty}^{\infty} a_{n} .
$$

Clearly $\|\pi\| \leq 1$. Moreover if $E$ is $J$-non-trivial, then $\pi:\left(\bigoplus \Delta_{m}(\bar{A})\right)_{E} \rightarrow$ $\bar{A}_{E ; J}$ is a metric surjection.

We will also need the diagonal operator

$$
j:\left(B_{0}, B_{1}\right) \rightarrow\left(\left(\bigoplus \Sigma_{m}(\bar{B})\right)_{\ell_{\infty}},\left(\bigoplus \Sigma_{m}(\bar{B})\right)_{\ell_{\infty}\left(2^{-m}\right)}\right)
$$

defined by

$$
j b=\{\ldots, b, b, b, \ldots\} \quad \text { for } b \in B_{0}+B_{1} .
$$

It is obvious that $j$ is bounded with $\|j\| \leq 1$ and $j: \bar{B}_{E ; K} \rightarrow\left(\bigoplus \Sigma_{m}(\bar{B})\right)_{E}$ is a metric injection whenever $E$ is $K$-non-trivial. 
Let $n \in \mathbb{N}$. Following [6] we define operators $P_{n}, Q_{n}^{+}, Q_{n}^{-}$on the Banach couple $\left(\left(\bigoplus \Delta_{m}(\bar{A})\right)_{\ell_{1}},\left(\bigoplus \Delta_{m}(\bar{A})\right)_{\ell_{1}\left(2^{-m}\right)}\right)$ by

$$
\begin{aligned}
P_{n}\left\{u_{m}\right\} & =\left\{\ldots, 0,0, u_{-n}, u_{-n+1}, \ldots, u_{n-1}, u_{n}, 0,0, \ldots\right\}, \\
Q_{n}^{+}\left\{u_{m}\right\} & =\left\{\ldots, 0,0, u_{n+1}, u_{n+2}, \ldots\right\}, \\
Q_{n}^{-}\left\{u_{m}\right\} & =\left\{\ldots, u_{-n-2}, u_{-n-1}, 0,0, \ldots\right\} .
\end{aligned}
$$

The following properties of the above operators are obvious:

- $I=P_{n}+Q_{n}^{+}+Q_{n}^{-}$, where $I$ denotes the identity operator on $\left(\bigoplus \Delta_{m}(\bar{A})\right)_{\ell_{1}}+\left(\bigoplus \Delta_{m}(\bar{A})\right)_{\ell_{1}\left(2^{-m}\right)}$.

- The operators $P_{n}, Q_{n}^{+}, Q_{n}^{-}, Q_{n}^{+}+Q_{n}^{-}$have norm 1 on $\left(\bigoplus \Delta_{m}(\bar{A})\right)_{\ell_{1}}$, $\left(\bigoplus \Delta_{m}(\bar{A})\right)_{\ell_{1}\left(2^{-m}\right)}$ and $\left(\bigoplus \Delta_{m}(\bar{A})\right)_{E}$.

- The following equalities hold:

$$
\begin{aligned}
\left\|Q_{n}^{+}\right\|_{\left(\oplus \Delta_{m}(\bar{A})\right)_{\ell_{1}} \rightarrow\left(\oplus \Delta_{m}(\bar{A})\right)_{\ell_{1}\left(2^{-m}\right)}} & =\left\|Q_{n}^{-}\right\|_{\left(\oplus \Delta_{m}(\bar{A})\right)_{\ell_{1}\left(2^{-m}\right)} \rightarrow\left(\oplus \Delta_{m}(\bar{A})\right)_{\ell_{1}}} \\
& =2^{-(n+1)}, \\
\left\|P_{n}\right\|_{\left(\oplus \Delta_{m}(\bar{A})\right)_{\ell_{1}} \rightarrow\left(\oplus \Delta_{m}(\bar{A})\right)_{\ell_{1}\left(2^{-m}\right)}} & =\left\|P_{n}\right\|_{\left(\oplus \Delta_{m}(\bar{A})\right)_{\ell_{1}\left(2^{-m}\right)} \rightarrow\left(\oplus \Delta_{m}(\bar{A})\right)_{\ell_{1}}} \\
& =2^{n} .
\end{aligned}
$$

In a similar way, we define the operators $R_{n}, S_{n}^{+}, S_{n}^{-}$on the Banach couple $\left(\left(\bigoplus \Sigma_{m}(\bar{B})\right)_{\ell_{\infty}},\left(\bigoplus \Sigma_{m}(\bar{B})\right)_{\ell_{\infty}\left(2^{-m}\right)}\right)$.

We recall that if $T: X \rightarrow Y$ is an operator, then the $n$th entropy number $\varepsilon_{n}(T):=\varepsilon_{n}(T: X \rightarrow Y), n \in \mathbb{N}$, is defined by

$$
\varepsilon_{n}(T)=\inf \left\{\varepsilon>0: T\left(B_{X}\right) \subset \bigcup_{i=1}^{n} B_{Y}\left(y_{i}, \varepsilon\right), y_{i} \in Y\right\},
$$

where for a given Banach space $Z$ and $\varepsilon>0$, we set $B_{Z}(x, \varepsilon):=\{y \in Z$ : $\left.\|x-y\|_{Z} \leq \varepsilon\right\}$ and $B_{Z}=B_{Z}(0,1)$.

We refer to [4] for the fundamental properties of the entropy numbers. The following facts are needed in what follows:

- $\varepsilon_{n}(T)>0$ for any $0 \neq T \in L(X, Y)$,

- $\|T\|=\varepsilon_{1}(T) \geq \varepsilon_{2}(T) \geq \cdots \geq 0$ for any $T \in L(X, Y)$,

- $\varepsilon_{k n}\left(T_{1}+T_{2}\right) \leq \varepsilon_{k}\left(T_{1}\right)+\varepsilon_{n}\left(T_{2}\right)$ for any $T_{1}, T_{2} \in L(X, Y)$,

- $\varepsilon_{k n}(R S) \leq \varepsilon_{k}(R) \varepsilon_{n}(S)$ for any $S \in L(X, Y), R \in L(Y, Z)$,

- $\varepsilon_{n}(T Q)=\varepsilon_{n}(T)$ for $T \in L(X, Y)$ and any metric surjection $Q: \widetilde{X} \rightarrow X$,

- $\varepsilon_{n}(T) \leq 2 \varepsilon_{n}(J T)$ for $T \in L(X, Y)$ and any metric injection $J: Y \rightarrow \widetilde{Y}$,

where $X, Y, Z, \widetilde{X}, \widetilde{Y}$ are arbitrary Banach spaces and $n, k \in \mathbb{N}$.

3. Main results. In this section, we prove our main results on estimates of the measure of non-compactness for special operators defined on Banach spaces and operators between Banach couples. We recall that the measure 
of non-compactness $\beta(T):=\beta(T: X \rightarrow Y)$ is defined for any bounded operator $T$ between Banach spaces by

$$
\beta(T):=\lim _{n \rightarrow \infty} \varepsilon_{n}(T) .
$$

In what follows, without further comment, we will use $C$ to denote positive constants ( $C$ may depend on objects that are regarded as fixed).

Theorem 3.1. Let $\bar{A}=\left(A_{0}, A_{1}\right)$ and $\bar{B}=\left(B_{0}, B_{1}\right)$ be Banach couples and let $T: \bar{A} \rightarrow \bar{B}$ be a bounded operator. Assume that $E$ is a parameter of the real method such that the Calderón operator $\Omega$ is bounded on E. Assume that

$$
\lim _{s \rightarrow 0+} \psi_{E}(s, 1)=\lim _{t \rightarrow 0+} \psi_{E}(1, t)=0 .
$$

Then there exists a constant $C=C(E)>0$ such that for any $\varepsilon>0$ and $k_{0}, k_{1} \in \mathbb{N}$, there exists $N \in \mathbb{N}$ such that for all $n>N$,

$$
\begin{aligned}
& \beta\left(\left(S_{n}^{+}+S_{n}^{-}\right) j T \pi\right.\left.\left(Q_{n}^{+}+Q_{n}^{-}\right):\left(\bigoplus \Delta_{m}(\bar{A})\right)_{E} \rightarrow\left(\bigoplus \Sigma_{m}(\bar{B})\right)_{E}\right) \\
& \leq C \psi_{E}\left(\varepsilon_{k_{0}}\left(T: A_{0} \rightarrow B_{0}\right)+\varepsilon, \varepsilon_{k_{1}}\left(T: A_{1} \rightarrow B_{1}\right)+\varepsilon\right) .
\end{aligned}
$$

Proof. Since for any $n \in \mathbb{N}$, we have

$$
\begin{aligned}
& \left(S_{n}^{+}+S_{n}^{-}\right) j T \pi\left(Q_{n}^{+}+Q_{n}^{-}\right) \\
& \quad=S_{n}^{+} j T \pi Q_{n}^{+}+S_{n}^{+} j T \pi Q_{n}^{-}+S_{n}^{-} j T \pi Q_{n}^{+}+S_{n}^{-} j T \pi Q_{n}^{-},
\end{aligned}
$$

it suffices to estimate the measure of non-compactness for each term.

First, note that for all $n \in \mathbb{N}$,

$$
\begin{aligned}
\left\|S_{n}^{+} j T \pi Q_{n}^{-}\right\|_{\left(\oplus \Delta_{m}(\bar{A})\right)_{\ell_{1}} \rightarrow\left(\oplus \Sigma_{m}(\bar{B})\right)_{\ell_{\infty}}} & \leq\|T\|_{A_{0} \rightarrow B_{0}}, \\
\left\|S_{n}^{-} j T \pi Q_{n}^{+}\right\|_{\left(\oplus \Delta_{m}(\bar{A})\right)_{\ell_{1}\left(2^{-m}\right)} \rightarrow\left(\oplus \Sigma_{m}(\bar{B})\right)_{\ell_{\infty}\left(2^{-m}\right)}} & \leq\|T\|_{A_{1} \rightarrow B_{1}} .
\end{aligned}
$$

Using the factorizations

$$
\begin{array}{ccc}
\left(\bigoplus \Delta_{m}(\bar{A})\right)_{\ell_{1}\left(2^{-m}\right)} \stackrel{S_{n}^{+} j T \pi Q_{n}^{-}}{\longrightarrow} & \left(\bigoplus \Sigma_{m}(\bar{B})\right)_{\ell_{\infty}\left(2^{-m}\right)} \\
Q_{n}^{-} \downarrow & \uparrow S_{n}^{+} \\
\left(\bigoplus \Delta_{m}(\bar{A})\right)_{\ell_{1}} \stackrel{j T \pi}{\longrightarrow} & \left(\bigoplus \Sigma_{m}(\bar{B})\right)_{\ell_{\infty}}
\end{array}
$$

and

$$
\begin{array}{ccc}
\left(\bigoplus \Delta_{m}(\bar{A})\right)_{\ell_{1}} & \stackrel{S_{n}^{-} j T \pi Q_{n}^{+}}{\longrightarrow} & \left(\bigoplus \Sigma_{m}(\bar{B})\right)_{\ell_{\infty}} \\
Q_{n}^{+} \downarrow & \uparrow S_{n}^{-} \\
\left(\bigoplus \Delta_{m}(\bar{A})\right)_{\ell_{1}\left(2^{-m}\right)} \stackrel{j T \pi}{\longrightarrow} & \left(\bigoplus \Sigma_{m}(\bar{B})\right)_{\ell_{\infty}\left(2^{-m}\right)}
\end{array}
$$


we obtain

$$
\begin{aligned}
\left\|S_{n}^{+} j T \pi Q_{n}^{-}\right\|_{\left(\oplus \Delta_{m}(\bar{A})\right)_{\ell_{1}\left(2^{-m}\right)} \rightarrow\left(\oplus \Sigma_{m}(\bar{B})\right)_{\ell_{\infty}\left(2^{-m}\right)}} \leq 2^{-2(n+1)}\|T\|_{A_{0} \rightarrow B_{0}}, \\
\left\|S_{n}^{-} j T \pi Q_{n}^{+}\right\|_{\left(\bigoplus \Delta_{m}(\bar{A})\right)_{\ell_{1}} \rightarrow\left(\oplus \Sigma_{m}(\bar{B})\right)_{\ell_{\infty}}} \leq 2^{-2(n+1)}\|T\|_{A_{1} \rightarrow B_{1}} .
\end{aligned}
$$

Combining the above estimates with Corollary 2.5 and Lemma 2.3, we find that there exists a constant $D=D(E)$ such that the measures of noncompactness of the operators $S_{n}^{+} j T \pi Q_{n}^{-}:\left(\bigoplus \Delta_{m}(\bar{A})\right)_{E} \rightarrow\left(\bigoplus \Sigma_{m}(\bar{B})\right)_{E}$ and $S_{n}^{-} j T \pi Q_{n}^{+}:\left(\bigoplus \Delta_{m}(\bar{A})\right)_{E} \rightarrow\left(\bigoplus \Sigma_{m}(\bar{B})\right)_{E}$ satisfy the following estimates:

$$
\begin{aligned}
\beta\left(S_{n}^{+} j T \pi Q_{n}^{-}\right) & \leq\left\|S_{n}^{+} j T \pi Q_{n}^{-}\right\| \leq D \psi_{E}\left(\|T\|_{A_{0} \rightarrow B_{0}}, 2^{-2(n+1)}\|T\|_{A_{0} \rightarrow B_{0}}\right) \\
& \leq D\|T\|_{A_{0} \rightarrow B_{0}} \psi_{E}\left(1,2^{-2(n+1)}\right),
\end{aligned}
$$

and

$$
\begin{aligned}
\beta\left(S_{n}^{-} j T \pi Q_{n}^{+}\right) & \leq D \psi_{E}\left(2^{-2(n+1)}\|T\|_{A_{1} \rightarrow B_{1}},\|T\|_{A_{1} \rightarrow B_{1}}\right) \\
& \leq D\|T\|_{A_{1} \rightarrow B_{1}} \psi_{E}\left(2^{-2(n+1)}, 1\right) .
\end{aligned}
$$

Now fix $\varepsilon>0$ and $k_{0}, k_{1} \in \mathbb{N}$, and put $\varepsilon_{k_{i}}(T)=\varepsilon_{k_{i}}\left(T: A_{i} \rightarrow B_{i}\right)$ for $i=0,1$. Note that there exists $N_{E} \in \mathbb{N}$ such that

$$
\beta\left(S_{n}^{+} j T \pi Q_{n}^{-}+S_{n}^{-} j T \pi Q_{n}^{+}\right) \leq D \psi_{E}\left(\varepsilon_{k_{0}}(T)+\varepsilon, \varepsilon_{k_{1}}(T)+\varepsilon\right)
$$

for all $n>N_{E}$.

In order to estimate the measures of non-compactness of the remaining two operators $S_{n}^{+} j T \pi Q_{n}^{+}$and $S_{n}^{-} j T \pi Q_{n}^{-}$, we first observe that the operators

$$
\begin{aligned}
& j T \pi Q_{n}^{+}:\left(\bigoplus \Delta_{m}(\bar{A})\right)_{\ell_{1}} \rightarrow\left(\bigoplus \Sigma_{m}(\bar{B})\right)_{\ell_{\infty}}, \\
& j T \pi Q_{n}^{-}:\left(\bigoplus \Delta_{m}(\bar{A})\right)_{\ell_{1}\left(2^{-m}\right)} \rightarrow\left(\bigoplus \Sigma_{m}(\bar{B})\right)_{\ell_{\infty}\left(2^{-m}\right)}
\end{aligned}
$$

are bounded and the sequences $\left\{\left\|j T \pi Q_{n}^{+}\right\|\right\}_{n=1}^{\infty},\left\{\left\|j T \pi Q_{n}^{-}\right\|\right\}_{n=1}^{\infty}$ of their norms are non-increasing. Let

$$
\lambda_{0}=\lim _{n \rightarrow \infty}\left\|j T \pi Q_{n}^{+}\right\|, \quad \lambda_{1}=\lim _{n \rightarrow \infty}\left\|j T \pi Q_{n}^{-}\right\|,
$$

and let $\left\{u_{m}^{0}\right\} \in B_{\left(\oplus \Delta_{m}(\bar{A})\right)_{\ell_{1}}}$ and $\left\{u_{m}^{1}\right\} \in B_{\left(\oplus \Delta_{m}(\bar{A})\right)_{\ell_{1}\left(2^{-m}\right)}}$ be sequences such that

$$
\begin{aligned}
& \lambda_{0}=\lim _{n \rightarrow \infty}\left\|j T \pi Q_{n}^{+} u_{n}^{0}\right\|_{\left(\oplus \Sigma_{m}(\bar{B})\right)_{\ell_{\infty}},} \\
& \lambda_{1}=\lim _{n \rightarrow \infty}\left\|j T \pi Q_{n}^{-} u_{n}^{1}\right\|_{\left(\oplus \Sigma_{m}(\bar{B})\right)_{\ell_{\infty}\left(2^{-m}\right)} .} .
\end{aligned}
$$

The properties of entropy numbers yield

$$
\begin{aligned}
& \varepsilon_{k_{0}}\left(j T \pi:\left(\bigoplus \Delta_{m}(\bar{A})\right)_{\ell_{1}}\right.\left.\rightarrow\left(\bigoplus \Sigma_{m}(\bar{B})\right)_{\ell_{\infty}}\right) \\
& \leq \varepsilon_{k_{0}}\left(T \pi:\left(\bigoplus \Delta_{m}(\bar{A})\right)_{\ell_{1}} \rightarrow B_{0}\right) \leq \varepsilon_{k_{0}}(T) \\
& \varepsilon_{k_{1}}\left(j T \pi:\left(\bigoplus \Delta_{m}(\bar{A})\right)_{\ell_{1}\left(2^{-m}\right)} \rightarrow\left(\bigoplus \Sigma_{m}(\bar{B})\right)_{\ell_{\infty}\left(2^{-m}\right)}\right) \\
& \leq \varepsilon_{k_{1}}\left(T \pi:\left(\bigoplus \Delta_{m}(\bar{A})\right)_{\ell_{1}\left(2^{-m}\right)} \rightarrow B_{1}\right) \leq \varepsilon_{k_{1}}(T) .
\end{aligned}
$$


From the definition of entropy numbers there exist sets $\left\{b_{1}^{0}, \ldots, b_{k_{0}}^{0}\right\} \subset B_{0}$ and $\left\{b_{1}^{1}, \ldots, b_{k_{1}}^{1}\right\} \subset B_{1}$ such that

$$
\begin{aligned}
T \pi\left(B_{\left(\oplus \Delta_{m}(\bar{A})\right)_{\ell_{1}}}\right) & \subset \bigcup_{j=1}^{k_{0}} B_{B_{0}}\left(b_{j}^{0}, \varepsilon_{k_{0}}(T)+\varepsilon / 2\right), \\
T \pi\left(B_{\left(\oplus \Delta_{m}(\bar{A})\right)_{\ell_{1}\left(2^{-m}\right)}}\right) & \subset \bigcup_{j=1}^{k_{1}} B_{B_{1}}\left(b_{j}^{1}, \varepsilon_{k_{1}}(T)+\varepsilon / 2\right) .
\end{aligned}
$$

Thus, for some $b_{j_{i}}^{i}, 1 \leq j_{i} \leq k_{i}$, and for some subsequences $\left\{n_{k}^{i}\right\}_{k=1}^{\infty}$ with $i=0,1$, we obtain

$$
\begin{aligned}
& T \pi Q_{n_{k}^{0}}^{+} u_{n_{k}^{0}}^{0} \in B_{B_{0}}\left(b_{j_{0}}^{0}, \varepsilon_{k_{0}}(T)+\varepsilon / 2\right), \\
& T \pi Q_{n_{k}^{1}}^{-} u_{n_{k}^{1}}^{1} \in B_{B_{1}}\left(b_{j_{1}}^{1}, \varepsilon_{k_{1}}(T)+\varepsilon / 2\right) .
\end{aligned}
$$

This implies

$$
\begin{aligned}
K\left(2^{m}, b_{j_{0}}^{0} ; \bar{B}\right) & \leq\left\|b_{j_{0}}^{0}-T \pi Q_{n_{k}^{0}}^{+} u_{n_{k}^{0}}^{0}\right\|_{B_{0}}+2^{m}\left\|T \pi Q_{n_{k}^{0}}^{+} u_{n_{k}^{0}}^{0}\right\|_{B_{1}} \\
& \leq\left(\varepsilon_{k_{0}}(T)+\varepsilon / 2\right)+2^{m}\|T\|_{A_{1} \rightarrow B_{1}} 2^{-\left(n_{k}^{0}+1\right)}\left\|u_{n_{k}^{0}}^{0}\right\|_{\left(\oplus \Delta_{m}(\bar{A})\right)_{\ell_{1}}} \\
& \leq\left(\varepsilon_{k_{0}}(T)+\varepsilon / 2\right)+2^{m-n_{k}^{0}}\|T\|_{A_{1} \rightarrow B_{1}}, \\
K\left(2^{m}, b_{j_{1}}^{1} ; \bar{B}\right) & \leq\left\|T \pi Q_{n_{k}^{1}}^{-} u_{n_{k}^{1}}^{1}\right\|_{B_{0}}+2^{m}\left\|b_{j_{1}}^{1}-T \pi Q_{n_{k}^{1}}^{-} u_{n_{k}^{1}}^{1}\right\|_{B_{1}} \\
& \leq\|T\|_{A_{0} \rightarrow B_{0}} 2^{-\left(n_{k}^{1}+1\right)}\left\|u_{n_{k}^{1}}^{1}\right\|_{\left(\oplus \Delta_{m}(\bar{A})\right)_{\ell_{1}\left(2^{-m}\right)}}+2^{m}\left(\varepsilon_{k_{1}}(T)+\varepsilon / 2\right) \\
& \leq 2^{-n_{k}^{1}}\|T\|_{A_{0} \rightarrow B_{0}}+2^{m}\left(\varepsilon_{k_{1}}(T)+\varepsilon / 2\right),
\end{aligned}
$$

and hence

$$
\begin{aligned}
\left\|j b_{j_{0}}^{0}\right\|_{\left(\oplus \Sigma_{m}(\bar{B})\right)_{\ell_{\infty}}} & =\sup _{m \in \mathbb{Z}} K\left(2^{m}, b_{j_{0}}^{0} ; \bar{B}\right) \leq \varepsilon_{k_{0}}(T)+\varepsilon / 2 \\
\left\|j b_{j_{1}}^{1}\right\|_{\left(\bigoplus \Sigma_{m}(\bar{B})\right)_{\ell_{\infty}\left(2^{-m}\right)}} & =\sup _{m \in \mathbb{Z}} 2^{-m} K\left(2^{m}, b_{j_{1}}^{1} ; \bar{B}\right) \leq \varepsilon_{k_{1}}(T)+\varepsilon / 2 .
\end{aligned}
$$

Consequently,

$$
\begin{aligned}
\lambda_{0} & =\lim _{k \rightarrow \infty}\left\|j T \pi Q_{n_{k}^{0}}^{+} u_{n_{k}^{0}}^{0}\right\|_{\left(\oplus \Sigma_{m}(\bar{B})\right)_{\ell_{\infty}}} \\
& \leq \sup _{k \in \mathbb{N}}\left(\left\|j T \pi Q_{n_{k}^{0}}^{+} u_{n_{k}^{0}}^{0}-j b_{j_{0}}^{0}\right\|_{\left(\oplus \Sigma_{m}(\bar{B})\right)_{\ell_{\infty}}}+\left\|j b_{j_{0}}^{0}\right\|_{\left(\oplus \Sigma_{m}(\bar{B})\right)_{\ell_{\infty}}}\right) \\
& \leq 2\left(\varepsilon_{k_{0}}(T)+\varepsilon / 2\right)
\end{aligned}
$$

and

$$
\begin{aligned}
\lambda_{1} & =\lim _{k \rightarrow \infty}\left\|j T \pi Q_{n_{k}^{1}}^{-} u_{n_{k}^{1}}^{1}\right\|_{\left(\oplus \Sigma_{m}(\bar{B})\right)_{\ell_{\infty}\left(2^{-m}\right)}} \\
& \leq \sup _{k \in \mathbb{N}}\left(\left\|j T \pi Q_{n_{k}^{1}}^{-} u_{n_{k}^{1}}^{1}-j b_{j_{1}}^{1}\right\|_{\left(\oplus \Sigma_{m}(\bar{B})\right)_{\ell_{\infty}\left(2^{-m}\right)}}+\left\|j b_{j_{1}}^{1}\right\|_{\left(\oplus \Sigma_{m}(\bar{B})\right)_{\ell_{\infty}\left(2^{-m}\right)}}\right) \\
& \leq 2\left(\varepsilon_{k_{1}}(T)+\varepsilon / 2\right) .
\end{aligned}
$$


Hence, there is $N_{a} \in \mathbb{N}$ such that for $n>N_{a}$,

$$
\begin{aligned}
\left\|S_{n}^{+} j T \pi Q_{n}^{+}\right\|_{\left(\oplus \Delta_{m}(\bar{A})\right)_{\ell_{1}} \rightarrow\left(\oplus \Sigma_{m}(\bar{B})\right)_{\ell_{\infty}}} & \leq 2\left(\varepsilon_{k_{0}}(T)+\varepsilon\right), \\
\left\|S_{n}^{-} j T \pi Q_{n}^{-}\right\|_{\left(\oplus \Delta_{m}(\bar{A})\right)_{\ell_{1}\left(2^{-m}\right)} \rightarrow\left(\oplus \Sigma_{m}(\bar{B})\right)_{\ell_{\infty}\left(2^{-m}\right)}} & \leq 2\left(\varepsilon_{k_{1}}(T)+\varepsilon\right) .
\end{aligned}
$$

With previously chosen $\varepsilon>0$, there exist sets $\left\{u_{1}^{0}, \ldots, u_{k_{0}}^{0}\right\} \subset B_{\left(\oplus \Delta_{m}(\bar{A})\right)_{\ell_{1}}}$ and $\left\{u_{1}^{1}, \ldots, u_{k_{1}}^{1}\right\} \subset B_{\left(\oplus \Delta_{m}(\bar{A})\right)_{\ell_{1}\left(2^{-m}\right)}}$ such that

$$
\begin{gathered}
j T \pi\left(B_{\left.\left(\oplus \Delta_{m}(\bar{A})\right)_{\ell_{1}}\right)} \subset \bigcup_{j=1}^{k_{0}} B_{\left(\oplus \Sigma_{m}(\bar{B})\right)_{\ell_{\infty}}\left(j T \pi u_{j}^{0}, 2 \varepsilon_{k_{0}}(T)+\varepsilon / 3\right),}\right. \\
j T \pi\left(B_{\left.\left(\oplus \Delta_{m}(\bar{A})\right)_{\ell_{1}\left(2^{-m}\right)}\right)} \subset \bigcup_{j=1}^{k_{1}} B_{\left(\oplus \Sigma_{m}(\bar{B})\right)_{\ell_{\infty}\left(2^{-m}\right)}}\left(j T \pi u_{j}^{1}, 2 \varepsilon_{k_{1}}(T)+\varepsilon / 3\right) .\right.
\end{gathered}
$$

Set $\delta_{i}=\varepsilon /\left(\|T\|_{A_{i} \rightarrow B_{i}}+\varepsilon\right)$ for $i=0,1$. There exists $N_{\varepsilon} \in \mathbb{N}$ such that

$$
\begin{aligned}
& \max _{1 \leq k \leq k_{0}}\left\|P_{N_{\varepsilon}} u_{k}^{0}-u_{k}^{0}\right\|_{\left(\oplus \Delta_{m}(\bar{A})\right)_{\ell_{1}}} \leq \delta_{0} / 3, \\
& \max _{1 \leq k \leq k_{1}}\left\|P_{N_{\varepsilon}} u_{k}^{1}-u_{k}^{1}\right\|_{\left(\oplus \Delta_{m}(\bar{A})\right)_{\ell_{1}(2-m)}} \leq \delta_{1} / 3 .
\end{aligned}
$$

This implies

$$
\begin{array}{r}
\max _{1 \leq k \leq k_{0}}\left\|j T \pi P_{N_{\varepsilon}} u_{k}^{0}-j T \pi u_{k}^{0}\right\|_{\left(\oplus \Sigma_{m}(\bar{B})\right)_{\ell_{\infty}}} \leq \varepsilon / 3, \\
\max _{1 \leq k \leq k_{1}}\left\|j T \pi P_{N_{\varepsilon}} u_{k}^{1}-j T \pi u_{k}^{1}\right\|_{\left(\oplus \Sigma_{m}(\bar{B})\right)_{\ell_{\infty}\left(2^{-m}\right)}} \leq \varepsilon / 3 .
\end{array}
$$

Thus for any $u^{0} \in B_{\left(\oplus \Delta_{m}(\bar{A})\right)_{\ell_{1}}}$ and any $u^{1} \in B_{\left(\oplus \Delta_{m}(\bar{A})\right)_{\ell_{1}\left(2^{-m}\right)}}$

$$
\begin{array}{r}
\min _{1 \leq k \leq k_{0}}\left\{\left\|j T \pi u^{0}-j T \pi P_{N_{\varepsilon}} u_{k}^{0}\right\|_{\left(\oplus \Sigma_{m}(\bar{B})\right)_{\ell_{\infty}}}\right\} \leq 2 \varepsilon_{k_{0}}(T)+2 \varepsilon / 3, \\
\min _{1 \leq k \leq k_{1}}\left\{\left\|j T \pi u^{1}-j T \pi P_{N_{\varepsilon}} u_{k}^{1}\right\|_{\left.\left(\oplus \Sigma_{m}(\bar{B})\right)_{\ell_{\infty}\left(2^{-m}\right)}\right\}} \leq 2 \varepsilon_{k_{1}}(T)+2 \varepsilon / 3 .\right.
\end{array}
$$

The diagrams

$$
\begin{array}{cc}
\left(\bigoplus \Delta_{m}(\bar{A})\right)_{\ell_{1}} \stackrel{S_{n}^{-} j T \pi P_{N_{\varepsilon}}}{\longrightarrow} & \left(\bigoplus \Sigma_{m}(\bar{B})\right)_{\ell_{\infty}} \\
P_{N_{\varepsilon}} \downarrow & \uparrow S_{n}^{-} \\
\left(\bigoplus \Delta_{m}(\bar{A})\right)_{\ell_{1}\left(2^{-m}\right)} \stackrel{j T \pi}{\longrightarrow} & \left(\bigoplus \Sigma_{m}(\bar{B})\right)_{\ell_{\infty}\left(2^{-m}\right)}
\end{array}
$$

and

$$
\begin{aligned}
& \left(\bigoplus \Delta_{m}(\bar{A})\right)_{\ell_{1}\left(2^{-m}\right)} \stackrel{S_{n}^{+} j T \pi P_{N_{\varepsilon}}}{\longrightarrow}\left(\bigoplus \Sigma_{m}(\bar{B})\right)_{\ell_{\infty}\left(2^{-m}\right)} \\
& P_{N_{\varepsilon}} \downarrow \\
& \left(\bigoplus \Delta_{m}(\bar{A})\right)_{\ell_{1}} \stackrel{j T \pi}{\longrightarrow} \quad\left(\bigoplus \Sigma_{m}(\bar{B})\right)_{\ell_{\infty}}
\end{aligned}
$$


imply that there exists $N_{b} \in \mathbb{N}$ such that for $n>N_{b}$,

$$
\begin{array}{r}
\max _{1 \leq k \leq k_{0}}\left\{\left\|S_{n}^{-} j T \pi P_{N_{\varepsilon}} u_{k}^{0}\right\|_{\left(\oplus \Sigma_{m}(\bar{B})\right)_{\ell_{\infty}}}\right\} \leq \varepsilon / 3, \\
\max _{1 \leq k \leq k_{1}}\left\{\left\|S_{n}^{+} j T \pi P_{N_{\varepsilon}} u_{k}^{1}\right\|_{\left(\oplus \Sigma_{m}(\bar{B})\right)_{\ell_{\infty}\left(2^{-m}\right)}}\right\} \leq \varepsilon / 3 .
\end{array}
$$

Thus for some $1 \leq j_{0} \leq k_{0}$ and $1 \leq j_{1} \leq k_{1}$, we obtain

$$
\begin{aligned}
\left\|S_{n}^{-} j T \pi u^{0}\right\|_{\left(\oplus \Sigma_{m}(\bar{B})\right)_{\ell_{\infty}} \leq} \leq & \left\|S_{n}^{-} j T \pi u^{0}-S_{n}^{-} j T \pi P_{N_{\varepsilon}} u_{j_{0}}^{0}\right\|_{\left(\oplus \Sigma_{m}(\bar{B})\right)_{\ell_{\infty}}} \\
& +\left\|S_{n}^{-} j T \pi P_{N_{\varepsilon}} u_{j_{0}}^{0}\right\|_{\left(\oplus \Sigma_{m}(\bar{B})\right)_{\ell_{\infty}}} \leq 2 \varepsilon_{k_{0}}(T)+\varepsilon
\end{aligned}
$$

and

$$
\begin{aligned}
\left\|S_{n}^{+} j T \pi u^{1}\right\|_{\left(\bigoplus \Sigma_{m}(\bar{B})\right)_{\ell_{\infty}\left(2^{-m}\right)} \leq} & \left\|S_{n}^{+} j T \pi u^{1}-S_{n}^{+} j T \pi P_{N_{\varepsilon}} u_{j_{1}}^{1}\right\|_{\left(\oplus \Sigma_{m}(\bar{B})\right)_{\ell_{\infty}\left(2^{-m}\right)}} \\
& +\left\|S_{n}^{+} j T \pi P_{N_{\varepsilon}} u_{j_{1}}^{1}\right\|_{\left(\oplus \Sigma_{m}(\bar{B})\right)_{\ell_{\infty}\left(2^{-m}\right)}} \\
\leq & 2 \varepsilon_{k_{1}}(T)+\varepsilon
\end{aligned}
$$

This implies

$$
\begin{aligned}
\left\|S_{n}^{-} j T \pi Q_{n}^{-}\right\|_{\left(\oplus \Delta_{m}(\bar{A})\right)_{\ell_{1} \rightarrow\left(\oplus \Sigma_{m}(\bar{B})\right)_{\ell_{\infty}}}} \leq 2 \varepsilon_{k_{0}}(T)+\varepsilon \\
\left\|S_{n}^{+} j T \pi Q_{n}^{+}\right\|_{\left(\oplus \Delta_{m}(\bar{A})\right)_{\ell_{1}\left(2^{-}-m\right)} \rightarrow\left(\oplus \Sigma_{m}(\bar{B})\right)_{\ell_{\infty}\left(2^{-m}\right)}} \leq 2 \varepsilon_{k_{1}}(T)+\varepsilon
\end{aligned}
$$

Consequently, we obtain

$$
\begin{aligned}
\beta\left(S_{n}^{+} j T \pi Q_{n}^{+}:\left(\bigoplus \Delta_{m}(\bar{A})\right)_{E}\right. & \left.\rightarrow\left(\bigoplus \Sigma_{m}(\bar{B})\right)_{E}\right) \\
\leq D \psi_{E}\left(2 \varepsilon_{k_{0}}(T)+2 \varepsilon, 2 \varepsilon_{k_{1}}(T)+2 \varepsilon\right) & \leq 2 D \psi_{E}\left(\varepsilon_{k_{0}}(T)+\varepsilon, \varepsilon_{k_{1}}(T)+\varepsilon\right) \\
\beta\left(S_{n}^{-} j T \pi Q_{n}^{-}:\left(\bigoplus \Delta_{m}(\bar{A})\right)_{E}\right. & \left.\rightarrow\left(\bigoplus \Sigma_{m}(\bar{B})\right)_{E}\right) \\
& \leq 2 D \psi_{E}\left(\varepsilon_{k_{0}}(T)+\varepsilon, \varepsilon_{k_{1}}(T)+\varepsilon\right) .
\end{aligned}
$$

Finally, the combination of the estimates obtained yields the required estimate

$$
\begin{gathered}
\beta\left(\left(S_{n}^{+}+S_{n}^{-}\right) j T \pi\left(Q_{n}^{+}+Q_{n}^{-}\right):\left(\bigoplus \Delta_{m}(\bar{A})\right)_{E} \rightarrow\left(\bigoplus \Sigma_{m}(\bar{B})\right)_{E}\right) \\
\leq C \psi_{E}\left(\varepsilon_{k_{0}}(T)+\varepsilon, \varepsilon_{k_{1}}(T)+\varepsilon\right)
\end{gathered}
$$

for all $n>N=\max \left\{N_{a}, N_{\varepsilon}, N_{b}, N_{E}\right\}$, where $C=C(E)>0$.

Theorem 3.2. Let $\bar{A}=\left(A_{0}, A_{1}\right)$ and $\bar{B}=\left(B_{0}, B_{1}\right)$ be Banach couples. Let $T: \bar{A} \rightarrow \bar{B}$ be a bounded operator and $E$ be a J-non-trivial $\mathbb{Z}$-lattice. Then for any $\varepsilon>0$ and $n, k_{0}, k_{1} \in \mathbb{N}$,

$$
\begin{aligned}
\beta\left(T \pi P_{n}\right)_{\left(\oplus \Delta_{m}(\bar{A})\right)_{E} \rightarrow \bar{B}_{E ; J}} & \leq 4 \psi_{E}\left(\varepsilon_{k_{0}}\left(T: A_{0} \rightarrow B_{0}\right)+\varepsilon, \varepsilon_{k_{1}}\left(T: A_{1} \rightarrow B_{1}\right)+\varepsilon\right) .
\end{aligned}
$$

Proof. The space $\left.E\right|_{[-n, n]}$ is finite-dimensional and $\left.E\right|_{[-n, n]} \stackrel{C}{\hookrightarrow} \ell_{\infty}^{(2 n+1)}$, where $C=C(n)>0$. Given any $\eta>0$, by the compactness argument there is a finite set $\left\{\mu_{1}, \ldots, \mu_{s}\right\} \subset B_{\left.E\right|_{[-n, n]}}$ such that for any $\lambda \in B_{\left.\left.E\right|_{[-n, n]}\right]}$, 


$$
\min _{1 \leq r \leq s}\left\{\left\|\lambda-\mu_{r}\right\|_{\left.E\right|_{[-n, n]}}\right\}<\eta / C .
$$

Take $\varepsilon>0$ and $k_{0}, k_{1} \in \mathbb{N}$. Let $2^{l_{i}-1} \leq \varepsilon_{k_{i}}\left(T: A_{i} \rightarrow B_{i}\right)+\varepsilon<2^{l_{i}}$ for $i=0,1$ and let $\nu=l_{1}-l_{0}$. For any $\left\{u_{m}\right\}_{m \in \mathbb{Z}} \in B_{\left(\oplus \Delta_{m}(\bar{A})\right)_{E}}$, we have

$$
\begin{aligned}
\left\|\left\{J\left(2^{-n}, u_{-n}\right), \ldots, J\left(2^{n}, u_{n}\right)\right\}\right\|_{\left.E\right|_{[-n, n]}} & =\left\|P_{n}\left\{u_{m}\right\}\right\|_{\left(\oplus \Delta_{m}(\bar{A})\right)_{E}} \\
& \leq\left\|\left\{u_{m}\right\}\right\|_{\left(\oplus \Delta_{m}(\bar{A})\right)_{E}} \leq 1 .
\end{aligned}
$$

Thus there exists a positive integer $r \leq s$ such that

$$
\max _{i=0,1}\left\{2^{i m}\left\|u_{m}\right\|_{A_{i}}\right\}=J\left(2^{m}, u_{m}\right)<\mu_{m}^{r}+\eta
$$

for $m=-n, \ldots, n$, where $\mu_{r}=\left\{\mu_{m}^{r}\right\}$. From the definition of $\varepsilon_{k_{i}}\left(T: A_{i} \rightarrow B_{i}\right)$, there exist finite sets $\left\{b_{1}^{i}, \ldots, b_{k_{i}}^{i}\right\} \subset B_{i}, i=0,1$, such that for $m=-n, \ldots, n$,

$$
\min _{1 \leq j \leq k_{i}}\left\{\left\|T u_{m}-\left(\mu_{m}^{r}+\eta\right) b_{j}^{i}\right\|_{B_{i}}\right\} \leq 2^{-i m}\left(\mu_{m}^{r}+\eta\right) 2^{l_{i}}=\eta_{r, m}^{i} .
$$

For $m \in\{-n, \ldots, n\}$ and positive integers $j_{0} \leq k_{0}, j_{1} \leq k_{1}$ and $r \leq s$ choose elements $d_{m}^{p}$ with $p=p\left(r, j_{0}, j_{1}\right)$ depending on $r, j_{0}, j_{1}$, where

$$
d_{m}^{p} \in B_{B_{0}}\left(\left(\mu_{m}^{r}+\eta\right) b_{j_{0}}^{0}, \eta_{r, m}^{0}\right) \cap B_{B_{1}}\left(\left(\mu_{m}^{r}+\eta\right) b_{j_{1}}^{1}, \eta_{r, m}^{1}\right),
$$

provided that the intersection is not empty. All elements $d_{m}^{p}$ are in $B_{0} \cap B_{1}$, and their number may change with $m$ (let us say it is $w=w(m)$ ), but it is finite. Given any $u_{m} \in B_{\left(\oplus \Delta_{m}(\bar{A})\right)_{E}}$, we can find a sequence $\left\{d_{m}^{p_{m}}\right\}_{m=-n}^{n}$, where $p_{m} \in[1, w(m)]$, such that for $m \in\{-n, \ldots, n\}$,

$$
\begin{aligned}
J\left(2^{m-\nu},\right. & \left.T u_{m}-d_{m}^{p_{m}}\right)=\max _{i=0,1}\left\{2^{i(m-\nu)}\left\|T u_{m}-d_{m}^{p_{m}}\right\|_{B_{i}}\right\} \\
& =\max _{i=0,1}\left\{2^{i(m-\nu)}\left\|T u_{m}-\left(\mu_{m}^{r}+\eta\right) b_{j_{i}}^{i}+\left(\mu_{m}^{r}+\eta\right) b_{j_{i}}^{i}-d_{m}^{p_{m}}\right\|_{B_{i}}\right\} \\
& \leq \max _{i=0,1}\left\{2 \eta_{r, m}^{0}, 2^{m-\nu} 2 \eta_{r, m}^{1}\right\}=2 \eta_{r, m}^{0} .
\end{aligned}
$$

Let $D_{J}$ be the collection of all sums $\sum_{m=-n}^{n} d_{m}^{p_{m}}$. Notice that $D_{J}$ is a finite subset of $\bar{B}_{E ; J}$ and for any $\left\{u_{m}\right\} \in B_{\left(\oplus \Delta_{m}(\bar{A})\right)_{E}}$ there exists one $\sum_{m=-n}^{n} d_{m}^{p_{m}} \in D_{J}$ such that

$$
\begin{aligned}
\| T \pi P_{n} & \left\{u_{m}\right\}-\sum_{m=-n}^{n} d_{m}^{p_{m}}\left\|_{\bar{B}_{E ; J}}=\right\| \sum_{m=-n}^{n}\left(T u_{m}-d_{m}^{p_{m}}\right) \|_{\bar{B}_{E ; J}} \\
& \leq\left\|\left\{J\left(2^{m}, T u_{m+\nu}-d_{m+\nu}^{p_{m+\nu}}\right)\right\}\right\|_{\left.E\right|_{[-n-\nu, n-\nu]}} \\
& \leq 2^{1+l_{0}}\left\|\left\{\mu_{m+\nu}^{r}+\eta\right\}\right\|_{\left.E\right|_{[-n-\nu, n-\nu]}} \leq 2^{1+l_{0}}\left\|\tau_{\nu}\right\|_{E \rightarrow E}\left\|\left\{\mu_{m}^{r}+\eta\right\}\right\|_{\left.E\right|_{[-n, n]}} \\
& \leq 2^{2+l_{0}-1}\left(1+\eta\left\|\sum_{m=-n}^{n} e_{m}\right\|_{E}\right)\left\|\tau_{l_{1}-1-l_{0}+1}\right\|_{E \rightarrow E} .
\end{aligned}
$$

Since $\varepsilon>0$ and $\eta$ can be arbitrarily small the required estimate follows. 
Theorem 3.3. Let $\bar{A}=\left(A_{0}, A_{1}\right)$ and $\bar{B}=\left(B_{0}, B_{1}\right)$ be Banach couples. Let $T: \bar{A} \rightarrow \bar{B}$ be a bounded operator and $E$ be a $K$-non-trivial $\mathbb{Z}$-lattice. Then for any $\varepsilon>0$ and $n, k_{0}, k_{1} \in \mathbb{N}$,

$$
\begin{aligned}
\beta\left(R_{n} j T: \bar{A}_{E ; K}\right. & \left.\rightarrow\left(\bigoplus \Sigma_{m}(\bar{B})\right)_{E}\right) \\
& \leq 4 \psi_{E}\left(\varepsilon_{k_{0}}\left(T: A_{0} \rightarrow B_{0}\right)+\varepsilon, \varepsilon_{k_{1}}\left(T: A_{1} \rightarrow B_{1}\right)+\varepsilon\right) .
\end{aligned}
$$

Proof. Fix $\varepsilon>0$ and $k_{0}, k_{1} \in \mathbb{N}$. Suppose that $2^{l_{i}-1} \leq \varepsilon_{k_{i}}\left(T: A_{i} \rightarrow B_{i}\right)+$ $\varepsilon<2^{l_{i}}$ for $i=0,1$ and let $\nu=l_{1}-l_{0}$. There exists a constant $C=C(n)>0$ such that $\left.E\right|_{[-n+\nu, n+\nu]} \stackrel{C}{\hookrightarrow} \ell_{\infty}^{2 n+1}$. Given any $\eta>0$, there exists a finite set $\left\{\mu_{1}, \ldots, \mu_{s}\right\} \subset B_{\left.E\right|_{[-n+\nu, n+\nu]}}$ such that for any $\lambda \in B_{\left.E\right|_{[-n+\nu, n+\nu]}}$,

$$
\min _{1 \leq r \leq s}\left\{\left\|\lambda-\mu_{r}\right\|_{\left.E\right|_{[-n+\nu, n+\nu]}}\right\}<\eta / C \text {. }
$$

For any $a \in B_{\bar{A}_{E ; K}}$, we have

$$
\left\|\left\{K\left(2^{-n+\nu}, a\right), \ldots, K\left(2^{n+\nu}, a\right)\right\}\right\|_{\left.E\right|_{[-n+\nu, n+\nu]}} \leq\left\|\left\{K\left(2^{m}, a\right)\right\}\right\|_{E}=\|a\|_{\bar{A}_{E ; K}} \leq 1 .
$$

Thus there is $\mu_{r}=\left\{\mu_{m}^{r}\right\}$, where $r \leq s$, such that

$$
K\left(2^{m+\nu}, a\right)<\mu_{m+\nu}^{r}+\eta \quad \text { for any } m \in\{-n, \ldots, n\} .
$$

From the definition of the $K$-functional, there exist decompositions

$$
a=a_{m}^{0}+a_{m}^{1}, \quad \text { where } a_{m}^{0} \in A_{0}, a_{m}^{1} \in A_{1},
$$

which yields

$$
\left\|a_{m}^{0}\right\|_{A_{0}}+2^{m+\nu}\left\|a_{m}^{1}\right\|_{A_{1}} \leq \mu_{m+\nu}^{r}+\eta
$$

for $m=-n, \ldots, n$. By definition of $\varepsilon_{k_{i}}\left(T: A_{i} \rightarrow B_{i}\right)$, there exist finite sets $\left\{b_{1}^{i}, \ldots, b_{k_{i}}^{i}\right\} \subset B_{i}, i=0,1$, such that

$$
\min _{1 \leq j \leq k_{i}}\left\{\left\|T a_{m}^{i}-\left(\mu_{m+\nu}^{r}+\eta\right) b_{j}^{i}\right\|_{B_{i}}\right\} \leq 2^{-i(m+\nu)}\left(\mu_{m+\nu}^{r}+\eta\right) 2^{l_{i}}=\eta_{r, m}^{i} .
$$

Denote by $D_{K}$ the collection of all vector-valued sequences $\left\{d_{m}^{p_{m}}\right\}$ defined by

$$
d_{m}^{p_{m}}= \begin{cases}\left(\mu_{m+\nu}^{r}+\eta\right)\left(b_{j_{m}^{0}}^{0}+b_{j_{m}^{1}}^{1}\right), & 1 \leq r \leq s, 1 \leq j_{m}^{0} \leq k_{0}, 1 \leq j_{m}^{1} \leq k_{1} \\ & \text { for } m \in\{-n, \ldots, n\} \\ 0, & \text { otherwise }\end{cases}
$$

where $p_{m}=p_{m}\left(r, j_{m}^{0}, j_{m}^{1}\right)$ depends on $r, j_{m}^{0}$ and $j_{m}^{1}$. Notice that $D_{K}$ is a finite subset of $\left(\bigoplus \Sigma_{m}(\bar{B})\right)_{E}$. For any $a \in B_{\bar{A}_{E ; K}}$, we can choose a sequence $\left\{d_{m}^{p_{m}}\right\} \in D_{K}$ with

$$
\left\|T a_{m}^{i}-\left(\mu_{m+\nu}^{r}+\eta\right) b_{j_{m}^{i}}^{i}\right\|_{B_{i}} \leq \eta_{r, m}^{i} \quad \text { for } i=0,1 .
$$

Thus 


$$
\begin{aligned}
K\left(2^{m}, T a\right. & \left.-\left(\mu_{m+\nu}^{r}+\eta\right)\left(b_{j_{m}^{0}}^{0}+b_{j_{m}^{1}}^{1}\right)\right) \\
& \left.=K\left(2^{m}, T a_{m}^{0}-\left(\mu_{m+\nu}^{r}+\eta\right) b_{j_{m}^{0}}^{0}+T a_{m}^{1}-\left(\mu_{m+\nu}^{r}+\eta\right) b_{j_{m}^{1}}^{1}\right)\right) \\
& \leq \eta_{r, m}^{0}+2^{m} \eta_{r, m}^{1}=2 \eta_{r, m}^{0} .
\end{aligned}
$$

Consequently,

$$
\begin{aligned}
\left\|R_{n} j T a-\left\{d_{m}^{p_{m}}\right\}\right\|_{\left(\oplus \Sigma_{m}(\bar{B})\right)_{E}} & =\|\left\{K\left(2^{m}, T a-\left(\mu_{m+\nu}^{r}+\eta\right)\left(b_{j_{m}^{0}}^{0}+b_{j_{m}^{1}}^{1}\right)\right\} \|_{\left.E\right|_{[-n, n]}}\right. \\
& \leq 2^{1+l_{0}}\left\|\left\{\mu_{m+\nu}^{r}+\eta\right\}\right\|_{\left.E\right|_{[-n, n]}} \\
& \leq 2^{1+l_{0}}\left\|\tau_{\nu}\right\|_{E \rightarrow E}\left\|\left\{\mu_{m}^{r}+\eta\right\}\right\|_{\left.E\right|_{[-n+\nu, n+\nu]}} \\
& \leq 2^{2+l_{0}-1}\left(1+\eta\left\|\sum_{m=-n+\nu}^{n+\nu} e_{m}\right\|_{E}\right)\left\|\tau_{l_{1}-1-l_{0}+1}\right\|_{E \rightarrow E}
\end{aligned}
$$

Since $\eta$ is arbitrary, the estimate follows.

ThEOREM 3.4. Let $\bar{A}=\left(A_{0}, A_{1}\right)$ and $\bar{B}=\left(B_{0}, B_{1}\right)$ be Banach couples. Let $T: \bar{A} \rightarrow \bar{B}$ be a bounded operator and $E$ be a parameter of the real method such that the Calderón transform $\Omega$ is a bounded operator on $E$. Assume that $\lim _{s \rightarrow 0+} \psi_{E}(s, 1)=\lim _{t \rightarrow 0+} \psi_{E}(1, t)=0$. Then there exists a constant $C=C(E)>0$ such that for any $\varepsilon>0$ and $k_{0}, k_{1} \in \mathbb{N}$,

$$
\beta\left(T: \bar{A}_{E} \rightarrow \bar{B}_{E}\right) \leq C \psi_{E}\left(\varepsilon_{k_{0}}\left(T: A_{0} \rightarrow B_{0}\right)+\varepsilon, \varepsilon_{k_{1}}\left(T: A_{1} \rightarrow B_{1}\right)+\varepsilon\right) .
$$

Proof. From the properties of the operators $\pi$ and $j$ we have

$$
\begin{aligned}
\beta\left(T: \bar{A}_{E ; J} \rightarrow \bar{B}_{E ; K}\right) & =\beta\left(T \pi:\left(\bigoplus \Delta_{m}(\bar{A})\right)_{E} \rightarrow \bar{B}_{E ; K}\right) \\
& \leq 2 \beta\left(j T \pi:\left(\bigoplus \Delta_{m}(\bar{A})\right)_{E} \rightarrow\left(\bigoplus \Sigma_{m}(\bar{B})\right)_{E}\right) .
\end{aligned}
$$

We decompose the operator $j T \pi$ as

$$
\begin{aligned}
j T \pi & =\left(R_{n}+S_{n}^{+}+S_{n}^{-}\right) j T \pi \\
& =R_{n} j T \pi+\left(S_{n}^{+}+S_{n}^{-}\right) j T \pi\left(P_{n}+Q_{n}^{+}+Q_{n}^{-}\right) \\
& =R_{n} j T \pi+\left(S_{n}^{+}+S_{n}^{-}\right) j T \pi P_{n}+\left(S_{n}^{+}+S_{n}^{-}\right) j T \pi\left(Q_{n}^{+}+Q_{n}^{-}\right)
\end{aligned}
$$

where $n \in \mathbb{N}$. Combining the results of Theorems 3.1 (with constant $D$ ), 3.2 and 3.3, we deduce that there exists $N \in \mathbb{N}$ such that for $n>N$,

$$
\begin{aligned}
& \beta\left(j T \pi:\left(\bigoplus \Delta_{m}(\bar{A})\right)_{E} \rightarrow\left(\bigoplus \Sigma_{m}(\bar{B})\right)_{E}\right) \\
& \leq\|\Omega\|_{E \rightarrow E} \beta\left(R_{n} j T: \bar{A}_{E ; K} \rightarrow\left(\bigoplus \Sigma_{m}(\bar{B})\right)_{E}\right) \\
&+\|\Omega\|_{E \rightarrow E} \beta\left(T \pi P_{n}:\left(\bigoplus \Delta_{m}(\bar{A})\right)_{E} \rightarrow \bar{B}_{E ; J}\right) \\
& \quad+\beta\left(\left(S_{n}^{+}+S_{n}^{-}\right) j T \pi\left(Q_{n}^{+}+Q_{n}^{-}\right):\left(\bigoplus \Delta_{m}(\bar{A})\right)_{E} \rightarrow\left(\bigoplus \Sigma_{m}(\bar{B})\right)_{E}\right) \\
& \leq\left(D+8\|\Omega\|_{E \rightarrow E}\right) \psi_{E}\left(\varepsilon_{k_{1}}\left(T: A_{0} \rightarrow B_{0}\right)+\varepsilon, \varepsilon_{k_{1}}\left(T: A_{1} \rightarrow B_{1}\right)+\varepsilon\right),
\end{aligned}
$$

which yields the result with a constant $C=C(E)>0$. 
We finish with some applications of the results obtained. The following is essentially a corollary of the properties of the measure of non-compactness and the results obtained above.

COROllary 3.5. Under the assumptions of Theorem 3.4,

$$
\beta\left(T: \bar{A}_{E} \rightarrow \bar{B}_{E}\right) \leq C \psi_{E}\left(\beta\left(T: A_{0} \rightarrow B_{0}\right), \beta\left(T: A_{1} \rightarrow B_{1}\right)\right) .
$$

In particular, if $T: A_{0} \rightarrow B_{0}$ or $T: A_{1} \rightarrow B_{1}$ is compact then $T: \bar{A}_{E} \rightarrow \bar{B}_{E}$ is also compact.

The compactness statement in Corollary 3.5 has been proved in $[5$, Thm. 5.4] via a direct proof without estimates of the measure of non-compactness.

Corollary 3.6. Let $\bar{A}=\left(A_{0}, A_{1}\right)$ and $\bar{B}=\left(B_{0}, B_{1}\right)$ be Banach couples and let $T: \bar{A} \rightarrow \bar{B}$ be an operator. If $\varrho$ is a function parameter, then there exists a constant $C>0$ such that whenever $\beta\left(T: A_{0} \rightarrow B_{0}\right)>0$, we have

$$
\beta\left(T: \bar{A}_{\varrho, q} \rightarrow \bar{B}_{\varrho, q}\right) \leq C \beta\left(T: A_{0} \rightarrow B_{0}\right) s_{\varrho}\left(\frac{\beta\left(T: A_{1} \rightarrow B_{1}\right)}{\beta\left(T: A_{0} \rightarrow B_{0}\right)}\right) .
$$

Proof. It is easy to see that $\left\|\tau_{n}\right\|_{E \rightarrow E} \leq s_{\varrho}\left(2^{n}\right)$ for $n \in \mathbb{Z}$, thus Corollary 3.5 applies.

REMARK 3.7. After the completion of this paper we were kindly informed by Fernando Cobos that the above estimate for the measure of noncompactness for interpolation spaces generated by a function parameter was proved independently by J. M. Cordeiro [8].

REMARK 3.8. We note that if $\varrho(t)=t^{\theta}$ with $\theta \in(0,1)$, then we recover the logarithmic type estimate for classical real interpolation spaces $\left(A_{0}, A_{1}\right)_{\theta, q}$ proved in [6]:

$$
\beta\left(T: \bar{A}_{\theta, q} \rightarrow \bar{B}_{\theta, q}\right) \leq C \beta\left(T: A_{0} \rightarrow B_{0}\right)^{1-\theta} \beta\left(T: A_{1} \rightarrow B_{1}\right)^{\theta}
$$

for some $C>0$.

REMARK 3.9. Let $A$ be any Banach space and $T$ be any bounded operator on $A$. It is well known (see, e.g., [4]) that the essential spectral radius of $T$ can be expressed in terms of the measure of non-compactness:

$$
r_{\mathrm{ess}}(T)=r_{\mathrm{ess}}(T: A \rightarrow A)=\lim _{n \rightarrow \infty} \beta\left(T^{n}: A \rightarrow A\right)^{1 / n} .
$$

The inequality

$$
\beta\left(T^{k n}: A \rightarrow A\right) \leq \beta\left(T^{k}: A \rightarrow A\right)^{n} \quad \text { for } k, n \in \mathbb{N}
$$

allows us to write

$$
\lim _{n \rightarrow \infty} \beta\left(T^{n}: A \rightarrow A\right)^{1 / n}=\inf _{1 \leq n<\infty} \beta\left(T^{n}: A \rightarrow A\right)^{1 / n} \leq \beta(T: A \rightarrow A) .
$$


We conclude the paper with the following result which extends previous results by Edmunds and Teixeira [15], Albrecht [1] and Cobos, FernándezMartínez and Martínez [6].

Corollary 3.10. Let $\bar{A}=\left(A_{0}, A_{1}\right)$ be a Banach couple of complex spaces. Assume that $T: \bar{A} \rightarrow \bar{A}$ is a bounded operator and $E$ is a parameter of the real method such that the Calderón transform $\Omega$ is a bounded operator on $E$ and

$$
\lim _{s \rightarrow 0+} \psi_{E}(s, 1)=\lim _{t \rightarrow 0+} \psi_{E}(1, t)=0 .
$$

Then

$$
r_{\mathrm{ess}}\left(T: A_{E} \rightarrow A_{E}\right) \leq C \psi_{E}\left(r_{\mathrm{ess}}\left(T: A_{0} \rightarrow A_{0}\right), r_{\mathrm{ess}}\left(T: A_{1} \rightarrow A_{1}\right)\right),
$$

with $C=C(E)>0$.

Proof. Applying Corollary 3.5, we obtain

$$
\begin{aligned}
\beta\left(T^{n}: \bar{A}_{E} \rightarrow \bar{A}_{E}\right) & \leq C \psi_{E}\left(\beta\left(T^{n}: A_{0} \rightarrow A_{0}\right), \beta\left(T^{n}: A_{1} \rightarrow A_{1}\right)\right) \\
& =C \psi_{E}\left(\left(\beta\left(T^{n}: A_{0} \rightarrow A_{0}\right)^{1 / n}\right)^{n},\left(\beta\left(T^{n}: A_{1} \rightarrow A_{1}\right)^{1 / n}\right)^{n}\right) \\
& \leq C C_{1}^{n-1} \psi_{E}\left(\beta\left(T^{n}: A_{0} \rightarrow A_{0}\right)^{1 / n}, \beta\left(T^{n}: A_{1} \rightarrow A_{1}\right)^{1 / n}\right)^{n}
\end{aligned}
$$

for any $n \in \mathbb{N}$. Therefore

$$
\begin{aligned}
\beta\left(T^{n}:\right. & \left.\bar{A}_{E} \rightarrow \bar{A}_{E}\right)^{1 / n} \\
& \leq C^{1 / n} C_{1}^{1-1 / n} \psi_{E}\left(\beta\left(T^{n}: A_{0} \rightarrow A_{0}\right)^{1 / n}, \beta\left(T^{n}: A_{1} \rightarrow A_{1}\right)^{1 / n}\right),
\end{aligned}
$$

which yields the result.

Acknowledgments. We thank the referee for his comments.

\section{References}

[1] E. Albrecht, Spectral interpolation, in Oper. Theory Adv. Appl. 14, Birkhäuser, Basel, 1984, 13-37.

[2] J. Bergh and J. Löfström, Interpolation Spaces, Springer, Berlin, 1976.

[3] Yu. A. Brudnyı̌ and N. Ya. Krugljak, Interpolation Functors and Interpolation Spaces, Vol. 1, North-Holland, Amsterdam, 1991.

[4] B. Carl and I. Stephani, Entropy, Compactness and the Approximation of Operators, Cambridge Univ. Press, 1990.

[5] F. Cobos, L. M. Fernández-Cabrera and A. Martínez, Compact operators between K- and J-spaces, Studia Math. 166 (2005), 199-220.

[6] F. Cobos, P. Fernández-Martínez, and A. Martínez, Interpolation of the measure of non-compactness by the real method, ibid. 135 (1999), 24-38.

[7] F. Cobos and J. Peetre, Interpolation of compactness using Aronszajn-Gagliardo functors, Israel J. Math. 68 (1989), 220-240.

[8] J. M. Cordeiro, Interpolación de ciertas clases de operadores por méthods multidimensionales, Publicatións do Dept. Mathemática Aplicada, Sec. 1, no. 9 (1999), Univesidade de Vigo. 
[9] M. Cwikel, Real and complex interpolation and extrapolation of compact operators, Duke Math. J. 65 (1992), 333-343.

[10] K. Hayakawa, Interpolation by the real method preserves compactness of operators, J. Math. Soc. Japan 21 (1969), 189-199.

[11] M. A. Krasnosel'skiı̌, On the theorem of M. Riesz, Soviet Math. Dokl. 1 (1960), 229-231.

[12] J. L. Lions et J. Peetre, Sur une classe d'espaces d'interpolation, Inst. Hautes Études Sci. Publ. Math. 19 (1964), 5-68.

[13] P. Nilsson, Reiteration theorems for real interpolation and approximation spaces, Ann. Mat. Pura Appl. 132 (1982), 291-330.

[14] A. Persson, Compact linear mappings between interpolation spaces, Ark. Mat. 5 (1964), 215-219.

[15] M. F. Teixeira and D. E. Edmunds, Interpolation theory and measure of noncompactness, Math. Nachr. 104 (1981), 129-135.

Faculty of Mathematics and Computer Science

A. Mickiewicz University

Umultowska 87

61-616 Poznań, Poland

E-mail: szwedek@amu.edu.pl

Received June 16, 2005

Revised version March 8, 2006 